(1)

CrossMark

\title{
NETs in pneumonia: is just enough the right amount?
}

\author{
Robert D. Gray (1) \\ Affiliation: MRC Centre for Inflammation Research at the University of Edinburgh, Queen's Medical Research \\ Institute, Edinburgh, UK. \\ Correspondence: Robert D. Gray, MRC Centre for Inflammation Research at the University of Edinburgh, \\ Queen's Medical Research Institute, Edinburgh, Scotland, UK. E-mail: r.d.graylaed.ac.uk
}

@ERSpublications

NETs have been suggested to have both detrimental and beneficial roles in lung infection and inflammation. The reality probably lies somewhere in the middle, where adequate NETs are needed to help control infection but excessive NETs cause damage http://ow.ly/IKH130jqFP3

Cite this article as: Gray RD. NETs in pneumonia: is just enough the right amount? Eur Respir J 2018; 51: 1800619 [https://doi.org/10.1183/13993003.00619-2018].

\begin{abstract}
Neutrophil extracellular traps (NETs) form when stimulated neutrophils undergo an oxidative burst and emit a complex of DNA, histones, pro-inflammatory and antibacterial proteins in a process distinct to other forms of neutrophil disposal such as apoptosis or necrosis. NETs have been associated with a number of important disease processes ranging from infectious diseases to sterile inflammatory conditions. This multi-potent role in immunity has led to conflicting opinion and evidence as to the beneficial or detrimental effects of NETs in immunity and indeed whether they have a major role in humans is as an antimicrobial defence [1]. In this issue of the European Respiratory Journal, EвRAHIMI et al. [2] demonstrate an association between high levels of nucleosomes (a marker of NETs) and adverse outcome in community-acquired pneumonia (CAP), in terms of time to patient stability, length of intravenous antibiotic treatment and time to discharge. Additionally, and perhaps more importantly, those with the highest levels of NETs had a higher (all cause) mortality. Interestingly, when the effects of corticosteroids on CAP outcome were investigated in this cohort, although these patients had a better clinical outcome they had higher persisting levels of NETs at days 5 and 7 (a feature also seen in the placebo arm of the trial where slower to decline levels of NETs were associated with better outcome), albeit they had started at a lower baseline. This presents a dichotomy where high levels of NETs (at presentation and over the course of CAP) are associated with bad outcome and death, whereas persisting lower levels of NETs into recovery are associated with better outcome. We might speculate that excessive levels of NETs in CAP could represent patients with a higher level of neutrophil activation in response to more severe infections or indeed patients with a higher level of systemic inflammation (a feature previously shown to be associated with adverse outcome in CAP [3]), but the clinical and pathological significance of slower to resolve NETs are less clear. Nevertheless, the present study suggests that the measurement of NETs may be a powerful biomarker of outcome in CAP. The authors go further in suggesting that excessive NETs at baseline and an early exhaustion of NET formation in the context of severe CAP is a potential mechanism leading to poor patient outcome; however, this remains speculative and further mechanistic studies will be required to investigate this feature. So, is there an optimal level of NETs at which health is maintained and too many or too few are detrimental?
\end{abstract}

Since the first description of NETs in 2004 their role as a potent mechanism to deal with invading pathogens has been suggested [4], and the fact that this process is highly conserved in the animal [5] and plant kingdom [6] has served to underline this. However, the overall contribution of NETs to bacterial 
killing in humans, in vivo, remains unclear, particularly in comparison to other processes such as phagocytosis. What is clear is that, even in that absence of additional antimicrobial therapies, the lung is highly capable of dealing with infectious insults, so much so that in the pre-antibiotic era the majority of hospitalised patients survived lobar pneumonia with a mortality of around 30\% [7]. Phagocytosis and the associated inflammation is a highly sophisticated response that has evolved to deal with the challenge of respiratory infections, and is still considered the main route for the clearance of bacteria and other pathogens from the lung. Recovery from infection (and the associated damage) is accompanied by resolution of the inflammatory response. Central to this process is the ability of neutrophils to undergo spontaneous apoptosis, which limits the release of histotoxic contents that may damage the surrounding lung tissue, and this is underlined by work demonstrating that lung damage caused by infection can be reduced by augmenting neutrophil apoptosis [8]. NET formation or NETosis may be considered an alternative form of neutrophil disposal (death), which is completely separate from apoptosis, and as such it may represent a pro-inflammatory form of cell death. How the balance between these modes of neutrophil disposal (and indeed others such as necroptosis) effects outcome is currently unknown.

Is there evidence that NETs are detrimental? Previous work has demonstrated a relationship between excessive NET formation and lung damage in an animal models of influenza [9], and decreasing NET levels can improve acute lung injury in mice infected with Gram-negative pathogens [10], consistent with the finding that acute respiratory distress syndrome patients with higher levels of plasma NETs had worse outcomes [10]. Taken together, these data suggest that higher levels of NET release may drive a damaging inflammatory response in the context of an infective lung insult. An additional consideration is how NETs may interact with other parts of the immune system and how this may have a bearing on disease processes. This is further underlined by the finding that levels of NETs are inversely correlated to lung function in cystic fibrosis (CF) [11], and disease severity in chronic obstructive pulmonary disease [12]. A major caveat to all of these studies is that they don't differentiate increased NET production from decreased NET clearance, which may be a crucial missing piece in the puzzle. Nevertheless, a growing body of evidence suggests that NETs are associated with worse outcomes in both acute and chronic respiratory disease, and this is further underlined by the work of EBRAHImi et al. [2].

Is there evidence that NETs are beneficial? In terms of an antimicrobial defence the ability of NETs to kill bacteria has been demonstrated to be independent of phagocytosis [4], but this is not a universal finding [13]. Evidence from animal models suggests that NET formation in response to bacteria may be dependent on pathogen type, with Staphylococcus aureus [14], Streptococcus pneumoniae [15] and Klebsiella pneumoniae [16] being associated with NET formation in murine models of lung infections. Conversely, a number of bacteria including S. pneumoniae, Haemophilus influenzae and Pseudomonas aeruginosa have developed evasion strategies to escape NET-mediated killing [17], thus further complicating our understanding of NET release in response to respiratory infection. The study of NETs in the context of respiratory infection has been hampered by the lack of specific tools to study the process in vivo, further complicated by the observation that NET release is a form of cell death termed NETosis, but in some scenarios it may occur as "vital NETosis" where nuclear material is released as a non-terminal event (for neutrophils) under specific circumstances [18]. The development of intravital microscopy to allow the study of infection and immune cell recruitment with high resolution in murine models of lung infection will facilitate the dissection of these mechanisms in vivo. This approach has recently been used to investigate the role of NETs in response to Gram negative and positive bacteria, and confirmed that increased levels of NETs are associated with lung damage. Interestingly, genetically knocking out NET formation led to bacterial dissemination from the lung and increased mortality, but controlling NETs to an intermediate level (by genetic modification or DNAse treatment) was associated with improved mortality [10]. These data support a major role for NETs in lung infection in the trapping of pathogens to prevent bacterial dissemination, as has also been suggested by previous studies [15], but this is clearly in fine balance with the potential of NETs to cause excessive inflammation and lung damage.

How do NETs cause inflammation? NETs have been associated with inflammation in a number of sterile inflammatory conditions such as systemic lupus erythematosus, rheumatoid arthritis, small vessel vasculitis, gout and cardiovascular disease [19], underlining a role for NETs in inflammation, although the role of NETs in the inflammatory response to infection is less well defined. Crucially the mechanisms by which NETs can promote inflammation are now being revealed and recent evidence suggests that the interaction of NETs with macrophages may be key to this [20, 21]. Therefore, NETs may have a crucial role to play in the crosstalk between cells of the innate (and adaptive) immune system, promoting inflammation, whereas other forms of neutrophil disposal such as apoptosis have a defined role in driving the resolution of inflammation [22]. Add to this the observation that NETs cause localised tissue damage independent of the infecting organism [23], and the potential for NETs as an inducer and perpetuator of inflammatory lung damage is apparent. A fine balance probably exists between an adequate amount of 
NETs to control infection and prime inflammation and excessive NETs causing local tissue damage and non-resolving inflammation.

EBRAHIMi et al. [2], although not uncovering a mechanistic role for NETosis in CAP, underline the potential importance of this process in what is a common respiratory illness associated with significant morbidity and mortality. The observation that corticosteroids lead to a prolonged lower level of NETs in recovering patients who have a better outcome does perhaps suggest a beneficial role for NETosis during the process of recovering from pneumonia, and would be in keeping with other recent data suggesting that patients who survive sepsis may indeed have a greater ability to make NETs than non-survivors [24]. Recent data from CF patients has suggested that a delay in neutrophil apoptosis in these subjects leads to an increased ability to form NETs [21], suggesting that the activation of alternative routes of disposal for neutrophils may have consequences in terms of promoting inflammation. Indeed the fact that corticosteroids are known to delay neutrophil apoptosis may be of direct consequence in the present study by preferentially allowing NETosis in those treated with prednisolone. More work is clearly required to probe this interesting area.

In conclusion, EBRAHimi et al. [2] contribute a clinical perspective to our current understanding of how NETs may be involved in pulmonary infection as well as potentially providing a new biomarker of outcome in CAP. Taken with other contemporary data it would appear that well controlled NET release and clearance are beneficial in processes such as pneumonia, but any imbalance in this system may be detrimental, with too many causing damaging inflammation or too few allowing dissemination of infection and sepsis. An increased focus on the interaction of NETs with other cells of the innate and adaptive immune system will be required to fully understand the immunological consequences of NET release in the context of infection, and how this process may be manipulated to improve disease outcomes. Going forward, specifically designed prospective studies focusing on the measurement of NETs during the evolution and resolution of pneumonia and associated inflammation in tandem with focused in vitro and in vivo experiments are essential to fully elucidate the mechanisms and consequences of NET release and clearance in CAP, and to definitively address what level of NET release is "just enough to be the right amount".

Conflict of interest: None declared.

\section{References}

$1 \quad$ Nauseef WM. Editorial: Nyet to NETs? A pause for healthy skepticism. J Leukoc Biol 2012; 91: 353-355.

2 Ebrahimi F, Giaglis S, Hahn S, et al. Markers of neutrophil extracellular traps predict adverse outcome in community-acquired pneumonia: secondary analysis of a randomised controlled trial. Eur Respir J 2018; 51: 1701389 .

3 Chalmers JD, Singanayagam A, Hill AT. C-reactive protein is an independent predictor of severity in community-acquired pneumonia. Am J Med 2008; 121: 219-225.

4 Brinkmann V, Reichard U, Goosmann C, et al. Neutrophil extracellular traps kill bacteria. Science 2004; 303: 1532-1535.

5 Robb CT, Dyrynda EA, Gray RD, et al. Invertebrate extracellular phagocyte traps show that chromatin is an ancient defence weapon. Nat Commun 2014; 5: 4627.

6 Tran TM, MacIntyre A, Hawes M, et al. Escaping underground nets: extracellular DNases degrade plant extracellular traps and contribute to virulence of the plant pathogenic bacterium Ralstonia solanacearum. PLoS Pathog 2016; 12: e1005686.

7 Heffron R. Pneumonia: with special reference to pneumococcus lobar pneumonia. New York, Commonwealth fund, 1939

8 Lucas CD, Dorward DA, Tait MA, et al. Downregulation of Mcl-1 has anti-inflammatory pro-resolution effects and enhances bacterial clearance from the lung. Mucosal Immunol 2014; 7: 857-868.

9 Narasaraju T, Yang E, Samy RP, et al. Excessive neutrophils and neutrophil extracellular traps contribute to acute lung injury of influenza pneumonitis. Am J Pathol 179: 199-210.

10 Lefrançais E, Mallavia B, Zhuo $\mathrm{H}$, et al. Maladaptive role of neutrophil extracellular traps in pathogen-induced lung injury. JCI Insight 2018; 3.

11 Marcos V, Zhou-Suckow Z, Önder Yildirim A, et al. Free DNA in cystic fibrosis airway fluids correlates with airflow obstruction. Mediators Inflamm 2015; 2015: 408935.

12 Dicker AJ, Crichton ML, Pumphrey EG, et al. Neutrophil extracellular traps are associated with disease severity and microbiota diversity in patients with chronic obstructive pulmonary disease. J Allergy Clin Immunol 2018; 141: 117-127.

13 Menegazzi R, Decleva E, Dri P. Killing by neutrophil extracellular traps: fact or folklore? Blood 2012; 119: 1214-1216.

14 Berends ET, Horswill AR, Haste NM, et al. Nuclease expression by Staphylococcus aureus facilitates escape from neutrophil extracellular traps. J Innate Immun 2010; 2: 576-586.

15 Beiter K, Wartha F, Albiger B, et al. An endonuclease allows Streptococcus pneumoniae to escape from neutrophil extracellular traps. Curr Biol CB 2006; 16: 401-407.

16 Papayannopoulos V, Metzler KD, Hakkim A, et al. Neutrophil elastase and myeloperoxidase regulate the formation of neutrophil extracellular traps. J Cell Biol 2010; 191: 677-691.

17 Storisteanu DML, Pocock JM, Cowburn AS, et al. Evasion of neutrophil extracellular traps by respiratory pathogens. Am J Respir Cell Mol Biol 2017; 56: 423-431. 
18 Pilsczek FH, Salina D, Poon KK, et al. A novel mechanism of rapid nuclear neutrophil extracellular trap formation in response to Staphylococcus aureus. J Immunol 2010; 185: 7413-7425.

19 Law SM, Gray RD. Neutrophil extracellular traps and the dysfunctional innate immune response of cystic fibrosis lung disease: a review. J Inflamm Lond Engl 2017; 14: 29.

20 Warnatsch A, Ioannou M, Wang Q, et al. Inflammation. Neutrophil extracellular traps license macrophages for cytokine production in atherosclerosis. Science 2015; 349: 316-320.

21 Gray RD, Hardisty G, Regan KH, et al. Delayed neutrophil apoptosis enhances NET formation in cystic fibrosis. Thorax 2018; 73: 134-144.

22 Rossi AG, Sawatzky DA, Walker A, et al. Cyclin-dependent kinase inhibitors enhance the resolution of inflammation by promoting inflammatory cell apoptosis. Nat Med 2006; 12: 1056-1064.

23 Kolaczkowska E, Jenne CN, Surewaard BGJ, et al. Molecular mechanisms of NET formation and degradation revealed by intravital imaging in the liver vasculature. Nat Commun 2015; 6: 6673.

24 Park SY, Shrestha S, Youn Y-J, et al. Autophagy primes neutrophils for neutrophil extracellular trap formation during sepsis. Am J Respir Crit Care Med 2017; 196: 577-589. 